$16^{\text {th }}$ International Conference on

AEROSPACE SCIENCES \& AVIATION TECHNOLOGY,

ASAT - 16 - May 26 - 28, 2015, E-Mail: asat@ mtc.edu.eg

Military Technical College, Kobry Elkobbah, Cairo, Egypt

Tel : +(202) 24025292 - 24036138, Fax: +(202) 22621908

\title{
Ethernet Wiring Standards with Different Frame Size to Enhance the Performance of LAN
}

\author{
Ashraf A.M. Khalaf*, Mostafa S.A. Mokadem ${ }^{\dagger}$ and Khalil A. Ahmad
}

\begin{abstract}
Commonly abbreviated Local Area Network LAN is a computer network that covers only a small area networks, such as campus computer networks, buildings, offices, in homes, schools or smaller. Currently, most LANs based on the IEEE 802.3 Ethernet technology using devices such as hubs and switches devices, which have a data transfer speed of 10, 100, or 1000 Mega bit per second ( Mbps). In this paper, we will evaluate the performance of LAN under different Ethernet wiring standards (10BaseT and 100BaseT) with different frame size.
\end{abstract}

Keywords: Frame size, 10BaseT, 100BaseT, LAN performance, Switch, Hub.

\section{Introduction}

A local area network (LAN) is a computer network that interconnects computers within limited area as computer laboratory or office building by using network media. In the implementation of LAN we use different types of devices as repeaters, switches, hubs, connectors and different cables.

LAN is found in many business environments that links a workgroup of task-related personal computers (PCs), for example, engineering workstations or accounting PCs. One of the computers is given a large capacity disk drive and become a server to all other PCs. Software can be stored on this server and used by the whole clients of the group. LAN covers only a small area networks (a few kilometers), such as campus computer networks, buildings, offices, in homes, schools or smaller. Currently, most LANs based on the IEEE 802.3 Ethernet technology using devices such as hubs and switches, which have a data transfer speed of 10, 100, or $1000 \mathrm{Mega}$ bit /s (Mbps). In the work done in [1], they are measuring the LAN performance. Their work depends on variation of the time of simulation and the number of hubs and making the frame size fixed value of (46, 2000 bytes) with segmentation (1500 bytes).

In the work done in [2], they are evaluating the performance of the LAN by varying the frame size between (1500, 1024 and 512) only and the variation of the Ethernet wiring standard.

The aim of this paper is to evaluate the performance of LANs in different Ethernet wiring standards (10BaseT and 100baseT) under different frame size (1500, 1024, 512, 128 and 64 bytes). Simulations are performed by using Riverbed Modeler Academic edition.

\footnotetext{
$\$$ Department of Power \& Machine Engineering, Faculty of Engineering, Minia University, Minia, khalilaa47@gmail.com

${ }^{\dagger}$ Engineer, Egyptian Electricity Holding Company, mostafashokry0@gmail.com

* Department of Electronics \& Communications Engineering, Faculty of Engineering, Minia University, ashkhalaf@yahoo.com
} 


\section{LAN Components}

\section{A. Network Media}

Network media, sometimes called Network medium, is the physical channel that used for transmission in network. There are two types of mediums used in the implementation of computer networks. One is guided medium and another is unguided medium [3], [4] and [5].

\section{Guided Media (wired)}

In guided medium electrical/optical signals are passed through a solid medium such as Copper Unshielded Twisted Pair (UTP), Copper shielded Twisted Pair (STP), Copper coaxial cables and fiber optics cables.in guided mediums, the signals are confined within the wire and do not propagate outside of the cables.

\section{Unguided Media (wireless)}

In unguided medium the data is transmitted by sending electromagnetic signals through free space and hence the signals are not guided in any specific direction. All unguided transmission mediums are classified as wireless transmission.

Table 1 shows the comparison between the guided cables (Twisted pair, Co-axial cables and optical fiber) [4].

Table 1 Comparison between guided cables

\begin{tabular}{l|l|l|l}
\hline \hline Media & Frequency range & Repeaters & Typical attenuation \\
\hline Twisted Pair & $0-3.5 \mathrm{KHz}$ & $2 \mathrm{Km}$ & $0.2 \mathrm{~dB} / \mathrm{km}$ at $1 \mathrm{KHz}$ \\
Coaxial Cable & $0-500 \mathrm{MHz}$ & $1-9 \mathrm{Km}$ & $7 \mathrm{~dB} / \mathrm{Km}$ at $10 \mathrm{MHz}$ \\
Optical Fibre & $186-370 \mathrm{THz}$ & $40 \mathrm{Km}$ & 0.2 to $0.5 \mathrm{~dB} / \mathrm{Km}$ \\
\hline
\end{tabular}

III. 10BaseT cables

It is a twisted pair Ethernet wiring standard for LAN implementation that support 10Mbps data rate. The maximum transmission length is 100 meters [5] and [7].

\section{100BaseT cables}

It is another twisted pair Ethernet wiring standard for LAN implementation that supports $100 \mathrm{Mbps}$ data rate. The 100BaseT Ethernet wiring standard is the most commonly used in LAN creation due to its high speed, robustness and low cost. It is also called fast Ethernet because it is ten times faster than 10BaseT [5], [7] and [9].

\section{B. HUB}

Hub is the simplest component in any local area network (LAN). Any data packet coming from one port is sent to all other ports it is then up to the receiving computer to decide if the packet is for it or not. Since every packet is sent out to every computer on the network there is a lot of wasted transmission, so the network can be easily become bogged down. Hubs are typically used on small networks where the amount of data going across the network is not very high [10]. 


\section{Switch}

Switch has multiple ports. When the packet comes through a switch it is read to determine which computer to send the data to. This leads to increase the efficiency and the performance of the device because the packets are not going to computers that do not require them [6].

\section{Simulation software and parameters}

The simulation will be done by using Riverbed Modeler Academic Edition 17.5 [8]. For this work we will create an office LAN which consists of hubs, switch, twenty Ethernet stations, 10 devices per each hub, under 10baseT (for scenario 1) and 100baseT (for scenario 2) Ethernet wiring standard.

\section{A. Riverbed Modeler Academic Edition 17.5}

Riverbed Modeler is software that is specialized for network research and development. This release replaced OPNET IT guru academic edition. I used that software to implement the office LAN because it offers relatively much powerful visual or graphical support for the users.

\section{B. Parameters of nodes}

\section{Traffic Generation Parameters}

Start time in seconds will be constant (5.0), ON State Time in second is constant (1000), OFF state Time is (0).

\section{Packet generation arguments}

Inter-arrival Time in seconds will be exponential (0.02), Packet size in bytes will be varied according to the frame size in each case which will be $(1500,1024,512,128,64)$, segmentation size in bytes will be No segmentation.

\section{Performance parameters}

I. For hub

Number of collision count, Utilization Traffic forwarded (bits/sec) and Traffic received (bits/sec).

\section{For Switch}

Traffic forwarded (bits/sec), Traffic received (bits/sec), Traffic filtered (bits/sec).

\section{Running time parameters}

The duration of the simulation will be $4 \mathrm{~min}$. 


\section{Simulation scenarios}

In our simulation we used two different scenarios for implementation of LANs with two different wiring Ethernet standard. At each scenario we changed the frame size to calculate some parameters of the network, then we evaluate the performance of the network.

\section{A. Scenario 1}

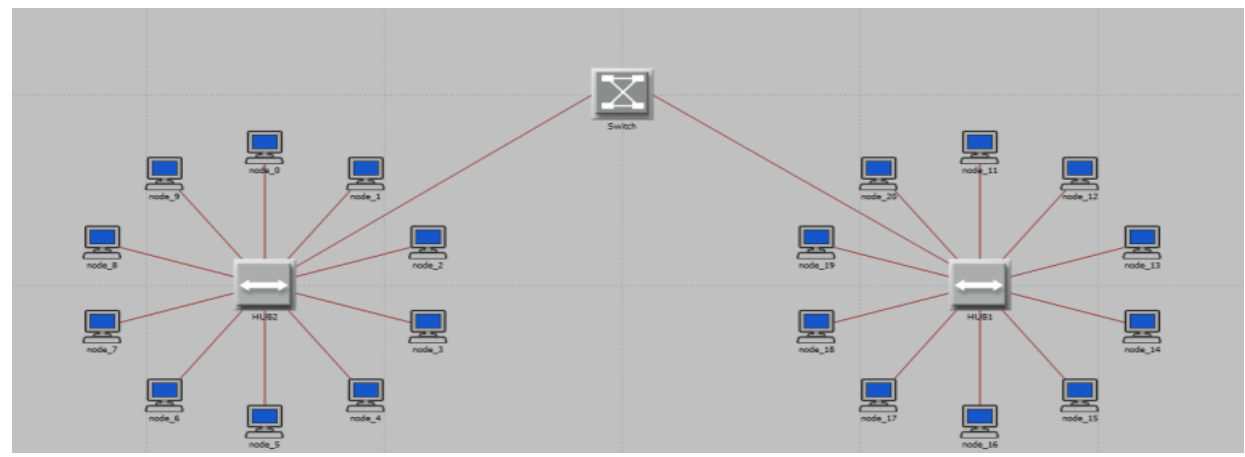

Fig. 1. Office LAN under 10BaseT wiring standard

Figure 1 illustrates scenario 1 which contains connection of 20 Ethernet stations to hubs, each hub connected to 10 Ethernet stations, and the hubs connected to Ethernet switch. 10BaseT Ethernet wiring standard will be used in that scenario.

\section{B. Scenario 2}

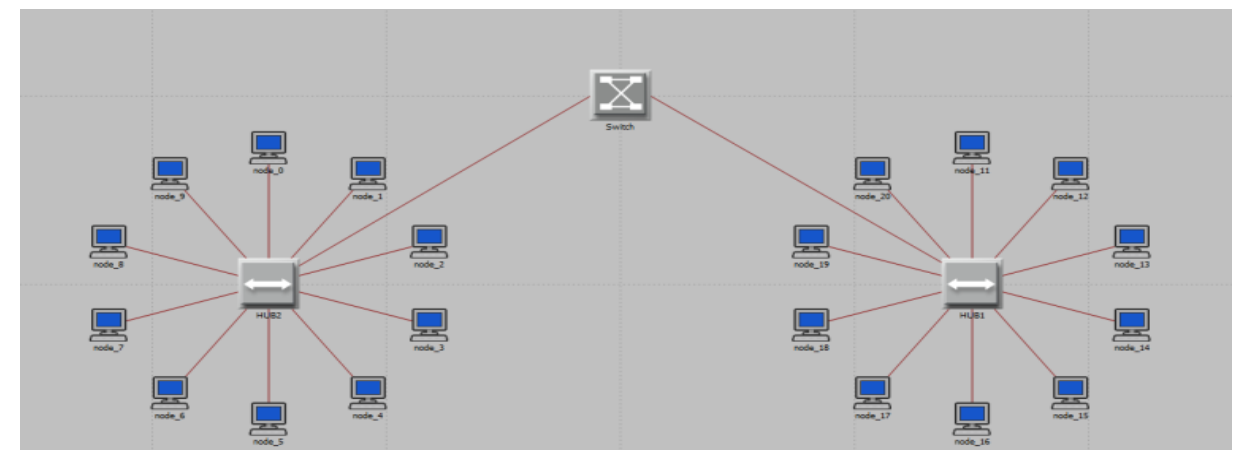

Fig. 2. Office LAN under 100BaseT wiring standard

Figure 2 illustrates scenario 2 which contains connection of 20 Ethernet stations to hubs, each hub connected to 10 Ethernet stations, and the hubs connected to Ethernet switch. 100BaseT Ethernet wiring standard will be used in that scenario.

\section{Simulation results}

After we made the simulation we took the results that measure and evaluate the performance of LAN under different Ethernet wiring standard with different frame size as following:

\section{A. Number of collision counts at Hub 1}


Table 2 Number of collision counts at HUB1 (Avg.)

\begin{tabular}{l|l|l}
\hline \hline Time duration & \multicolumn{2}{|c}{ 4 minutes } \\
\hline & \multicolumn{2}{|c}{ Collision count } \\
\hline Devices & \multicolumn{2}{|c}{ Hub 1 } \\
\hline standards & $\begin{array}{c}\text { 10BaseT } \\
\text { (scenario1) }\end{array}$ & $\begin{array}{l}\text { 100BaseT } \\
\text { (scenario2) }\end{array}$ \\
\hline 1500 bytes & $3,456.19$ & 24.24 \\
1024 bytes & $1,558.101$ & 14.051 \\
512 bytes & 333.03 & 7.753 \\
128 bytes & 26.292 & 5.589 \\
64 bytes & 13.54 & 4.7 \\
\hline \hline
\end{tabular}

Table 2 shows the comparison between the collision count number at hub 1 under 10BaseT (scenario 1) and 100BaseT (scenario 2) for 1500, 1024, 512, 128 and 64 bytes of frame size.

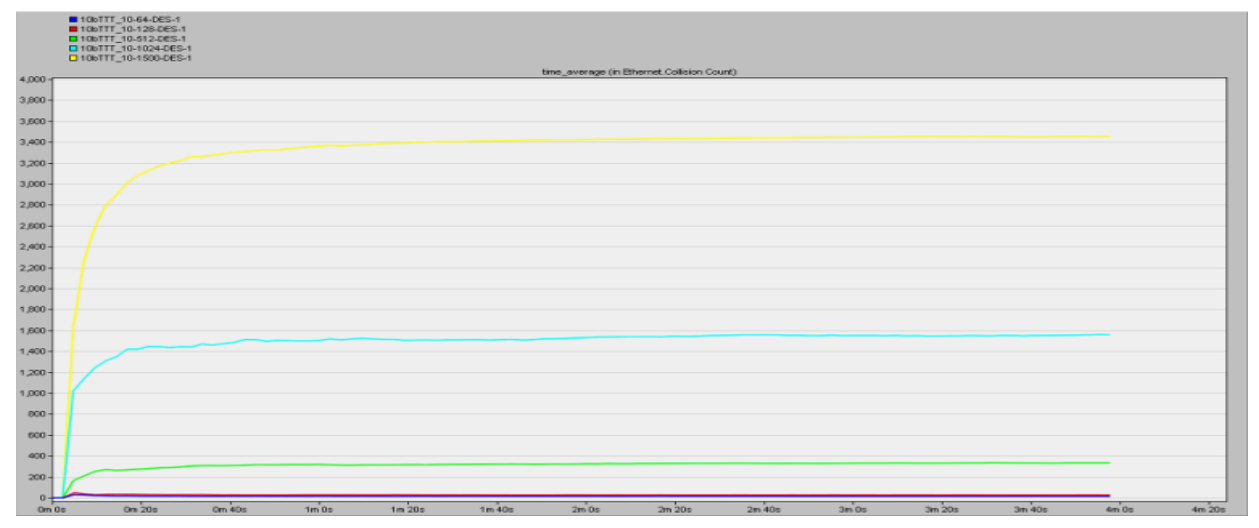

Fig. 3. Comparison between numbers of collision count at Hub1 under different frame size at 10BaseT Ethernet wiring standard.

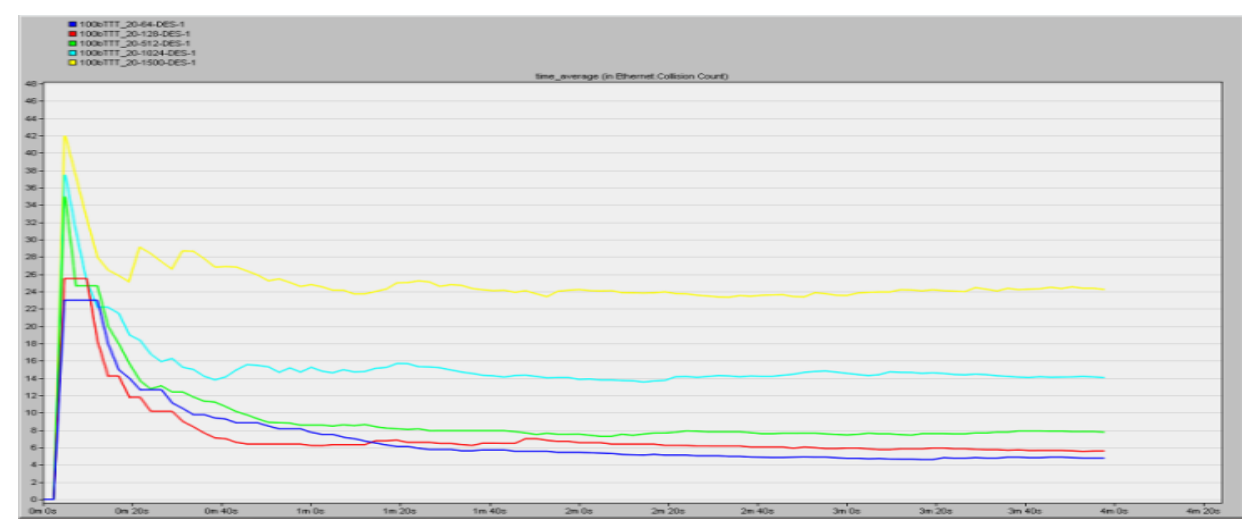

Fig. 4. Comparison between numbers of collision count at Hub1 under different frame size at 100BaseT Ethernet wiring standard 


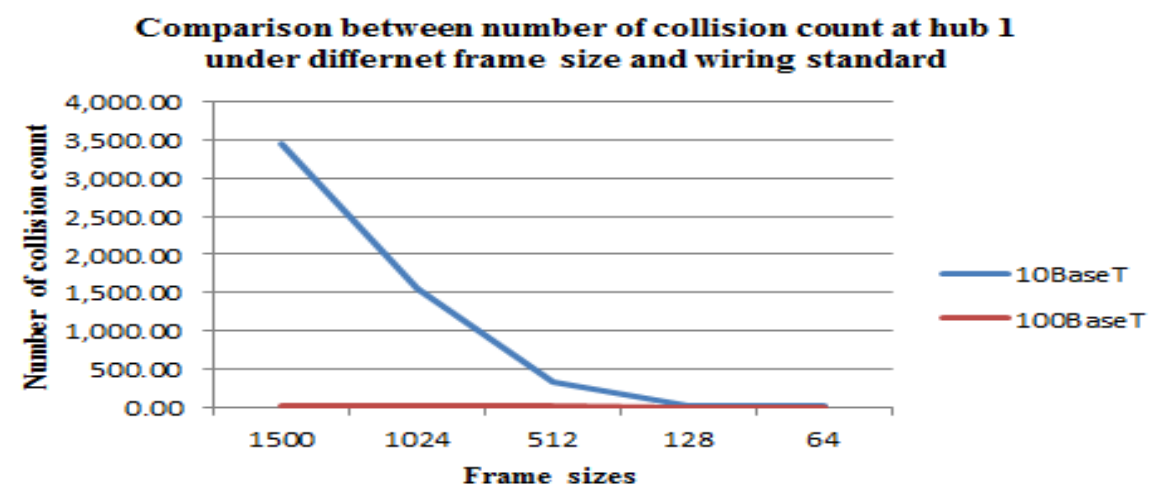

Fig. 5. Graphs for number of collision count at Hub1

Figures 3, 4 and 5 show that the number of collision counts in 10BaseT is more than 100BaseT for all frame sizes.

\section{B. Utilization of Hub 1}

Table 3 Utilization of Hub1 (Avg.)

\begin{tabular}{c|l|c}
\hline \hline Time duration & \multicolumn{2}{|c}{ 4 minutes } \\
\hline Devices & \multicolumn{2}{|c}{ Utilization } \\
\hline Standards & $\begin{array}{c}\text { 10BaseT } \\
\text { (scenario1) }\end{array}$ & $\begin{array}{c}100 \text { BaseT } \\
\text { (scenario2) }\end{array}$ \\
\hline 1500 bytes & 0.883 & 0.091 \\
1024 bytes & 0.627 & 0.062 \\
512 bytes & 0.321 & 0.032 \\
128 bytes & 0.092 & 0.009 \\
64 bytes & 0.053 & 0.005 \\
\hline \hline
\end{tabular}

Table 3 shows the comparison between the utilization of hub 1 under 10BaseT (scenario1) and 100BaseT (scenario2) for 1500, 1024, 512, 128 and 64 bytes of frame size.

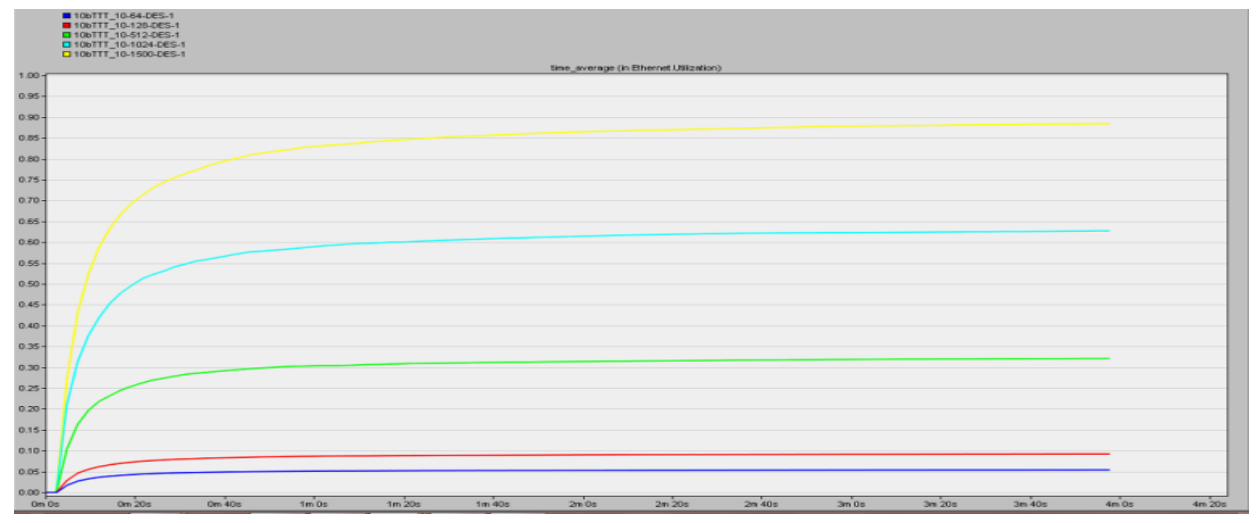

Fig. 6. Comparison between utilization at Hub1 under different frame size at 10BaseT Ethernet wiring standard. 


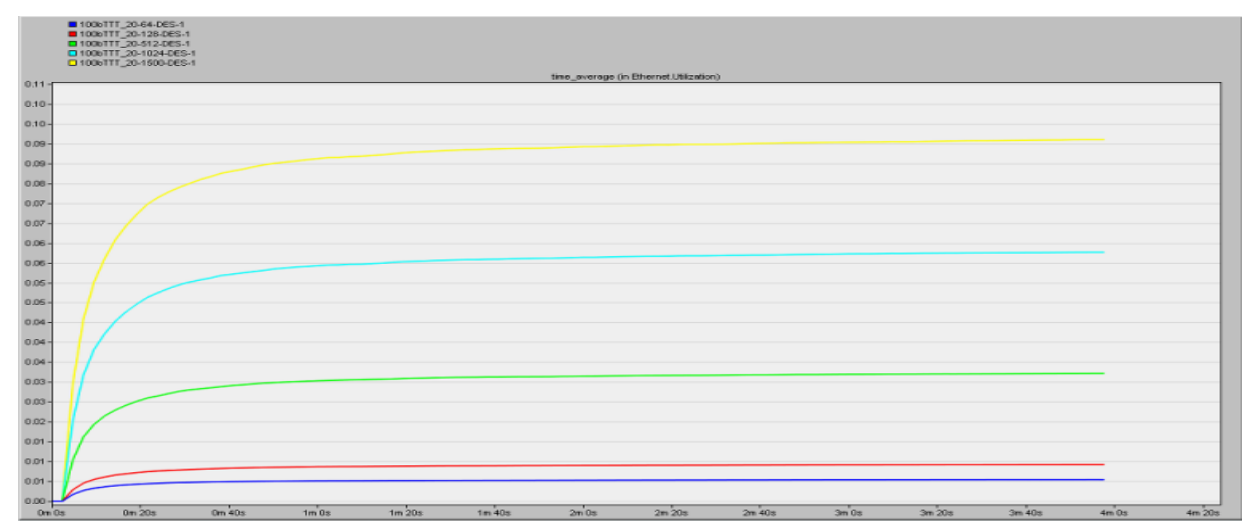

Fig. 7. Comparison between utilization at Hub1 under different frame size at 100BaseT Ethernet wiring standard

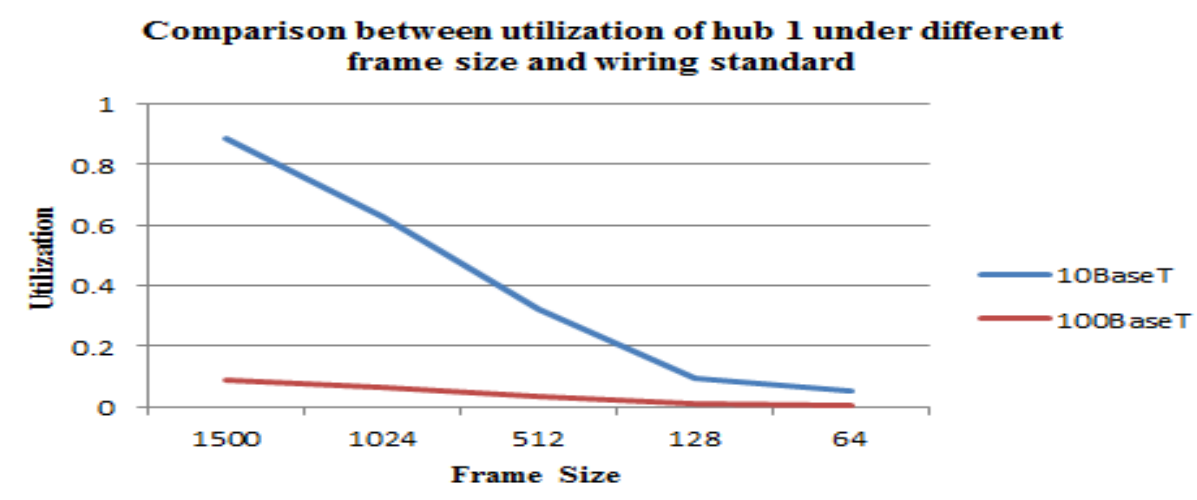

Fig. 8. Graphs for Utilization of Hub1

Figures 6, 7 and 8 demonstrate that the utilization in 10BaseT is more than 100BaseT for all frame sizes.

C. Traffic forwarded (bits/sec) at Hub1

Table 4 Traffic forwarded (bits/sec) at hub1 (Avg.)

\begin{tabular}{c|l|l}
\hline \hline Time duration & \multicolumn{2}{|c}{ 4 minutes } \\
\hline \multicolumn{1}{c|}{ Devices } & \multicolumn{2}{|c}{ Traffic Forwarded (bps) } \\
\hline \multicolumn{1}{c|}{ Standards } & \multicolumn{1}{|c}{$\begin{array}{c}\text { 10BaseT } \\
\text { (scenario1) }\end{array}$} & $\begin{array}{c}\text { 100BaseT } \\
\text { (scenario2) }\end{array}$ \\
\hline 1500 bytes & $8,815,602$ & $9,100,506$ \\
1024 bytes & $6,259,192$ & $6,263,042$ \\
512 bytes & $3,209,531$ & $3,214,534$ \\
128 bytes & 919,988 & 918,550 \\
64 bytes & 538,601 & 538,967 \\
\hline \hline
\end{tabular}

Table 4 shows the comparison between the traffic forwarded to Hub 1 under 10BaseT (scenario1) and 100BaseT (scenario2) for 1500, 1024, 512, 128 and 64 bytes of frame size. 


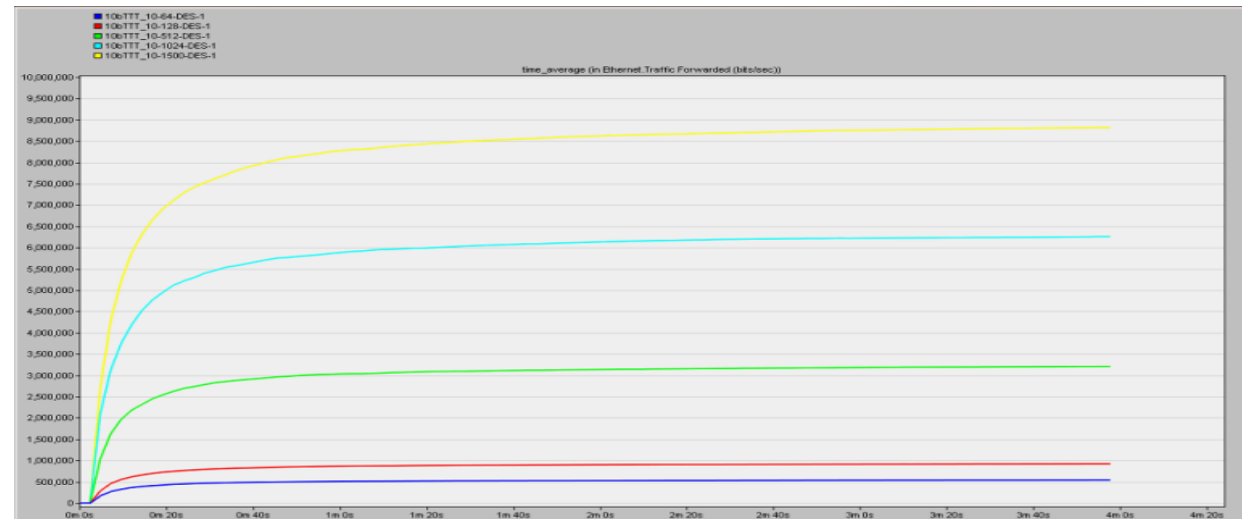

Fig. 9. Comparison between traffic forwarded (bps) at Hub1 under different frame size at 10BaseT Ethernet wiring standard

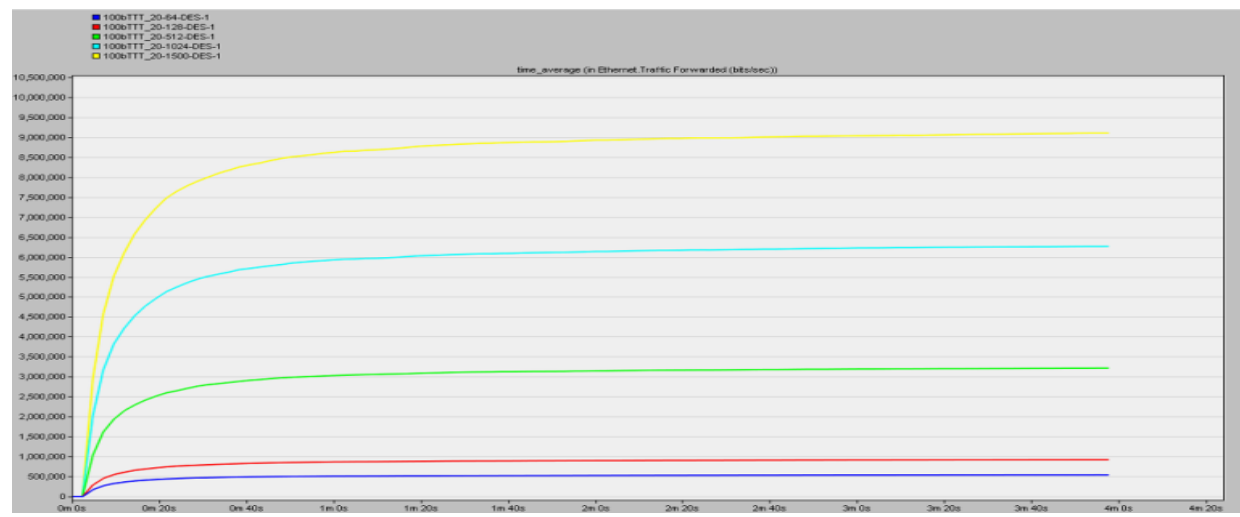

Fig. 10. Comparison between traffic forwarded (bps) at Hub1 under different frame size at 100BaseT Ethernet wiring standard

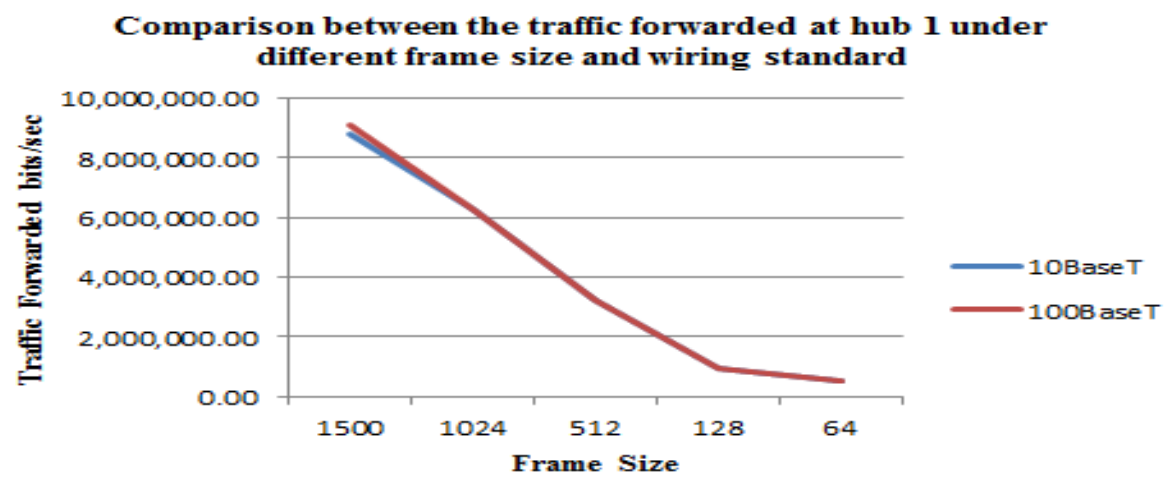

Fig. 11. Graphs of traffic forwarded at Hub1 (bit/sec)

Figures 9, 10 and 11 show that at some points both curves overlap to each other; it means that traffic forwarded to hub1 is approximately same at these points. 


\section{Traffic received (bits/sec) at Hub 1}

Table 5 Traffic received (bits/sec) at hub1 (Avg.)

\begin{tabular}{c|l|l}
\hline \hline Time duration & \multicolumn{2}{|c}{4 minutes } \\
\hline Devices & \multicolumn{2}{|c}{ Traffic Received (bps) } \\
\hline Standards & $\begin{array}{c}\text { 10BaseT } \\
\text { (scenario1) }\end{array}$ & $\begin{array}{c}\text { 100BaseT } \\
\text { (scenario2) }\end{array}$ \\
\hline 1500 bytes & $8,815,602$ & $9,100,506$ \\
1024 bytes & $6,259,192$ & $6,263,042$ \\
512 bytes & $3,209,531$ & $3,214,534$ \\
128 bytes & 919,988 & 918,550 \\
64 bytes & 538,601 & 538,967 \\
\hline \hline
\end{tabular}

Table 5 shows the comparison between the traffic received to Hub 1 under 10BaseT (scenario1) and 100BaseT (scenario2) for 1500, 1024, 512, 128 and 64 bytes of frame size.

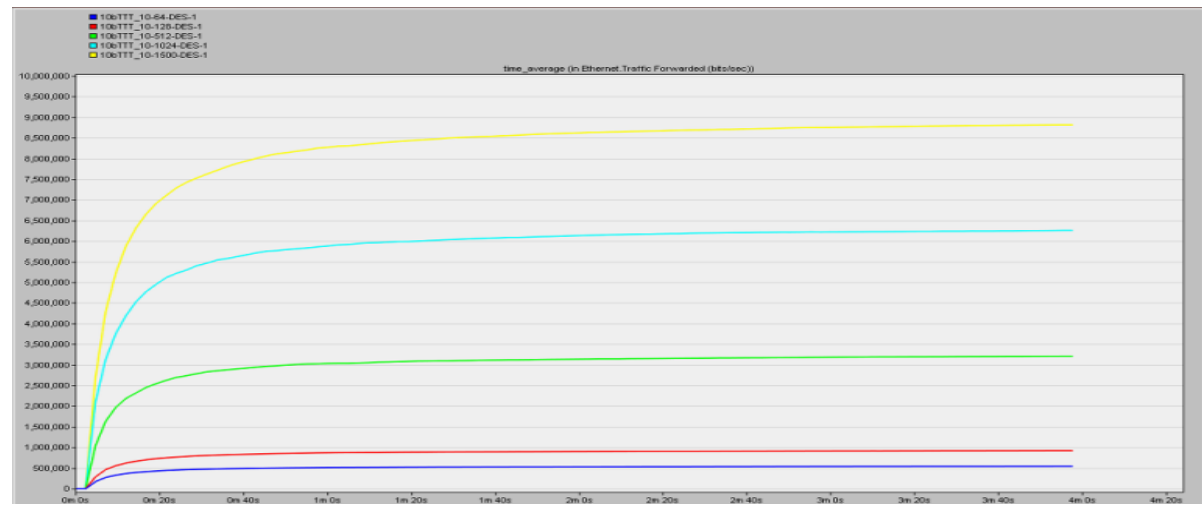

Fig. 12. Comparison between traffic received (bps) at Hub1 under different frame size at 10BaseT Ethernet wiring standard

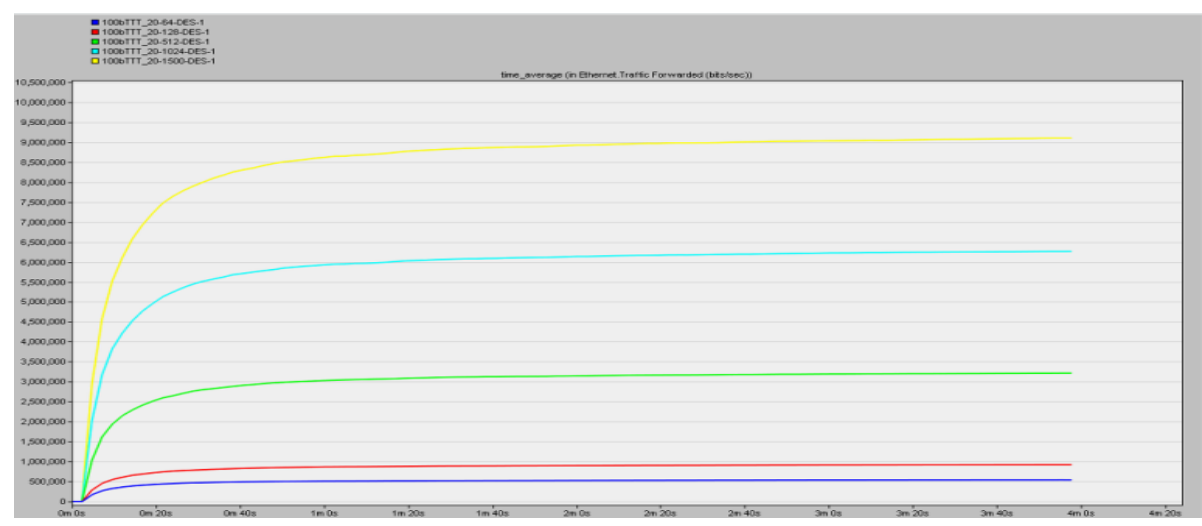

Fig. 13. Comparison between traffic forwarded (bps) at Hub1 under different frame size at 100BaseT Ethernet wiring standard 


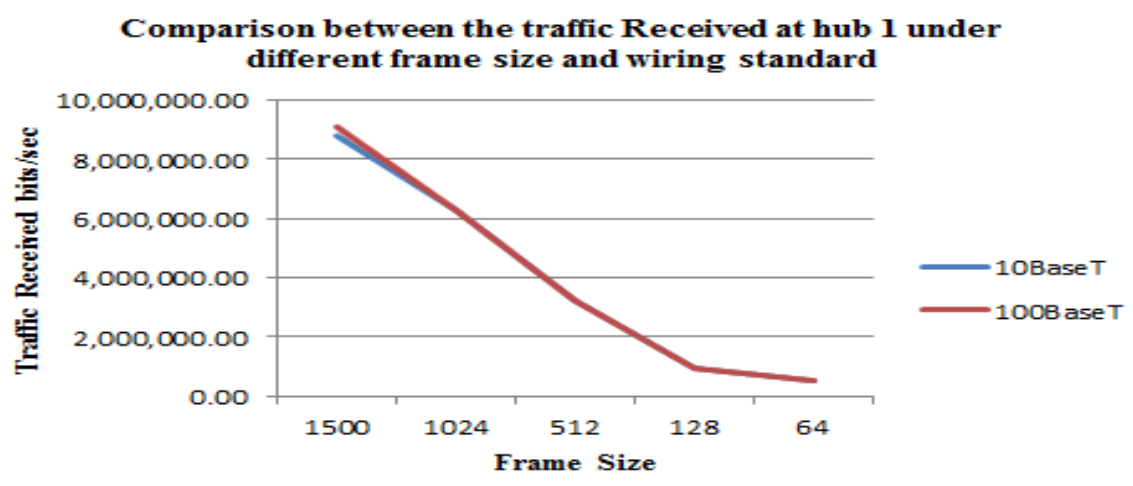

Fig. 14. Graphs of traffic received at hub1 (bit/sec)

Figures 12, 13 and 14 demonstrate that at some points both curves overlap to each other; it means that traffic received to hub1 is approximately same at these points.

E. Number of collision counts at Hub 2

Table 6 Number of collision counts at HUB2 (Avg.)

\begin{tabular}{c|l|l}
\hline \hline Time duration & \multicolumn{2}{|c}{ 4 minutes } \\
\hline Devices & \multicolumn{2}{|c}{ Collision count } \\
\hline Standards & $\begin{array}{c}\text { 10BaseT } 2 \\
\text { (scenario1) }\end{array}$ & $\begin{array}{c}\text { 100BaseT } \\
\text { (scenario2) }\end{array}$ \\
\hline 1500 bytes & $3,435.919$ & 27.70 \\
1024 bytes & $1,572.666$ & 14.877 \\
512 bytes & 340.3 & 7.626 \\
128 bytes & 29.4 & 5.29 \\
64 bytes & 12.4 & 5.13 \\
\hline
\end{tabular}

Table 6 illustrates the comparison between the collision count number at hub 2 under 10BaseT (scenario 1) and 100BaseT (scenario 2) for 1500, 1024, 512, 128 and 64 bytes of frame size.

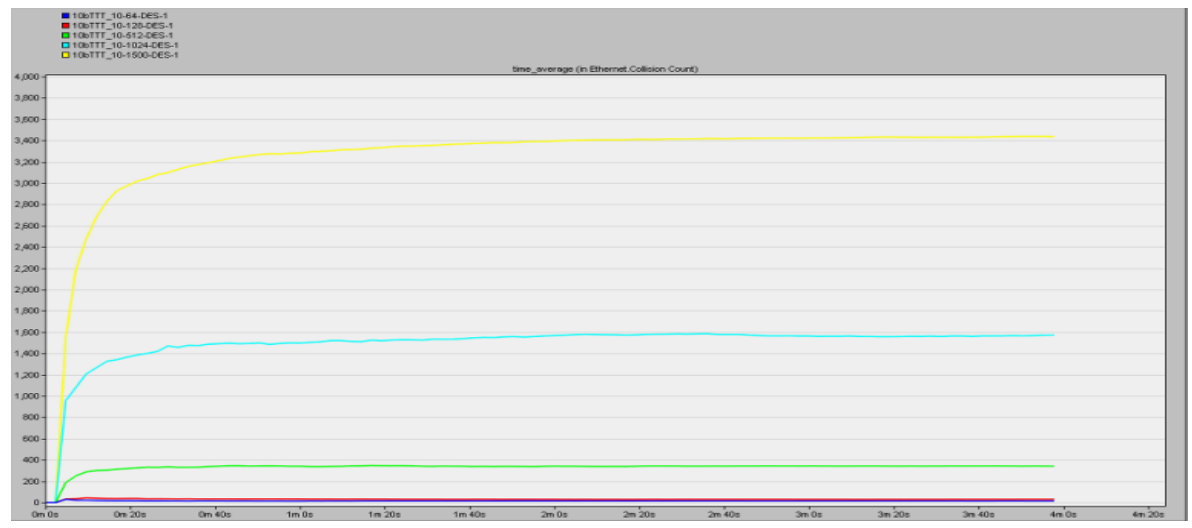

Fig. 15. Comparison between numbers of collision count at Hub2 under different frame size at 10BaseT Ethernet wiring standard 


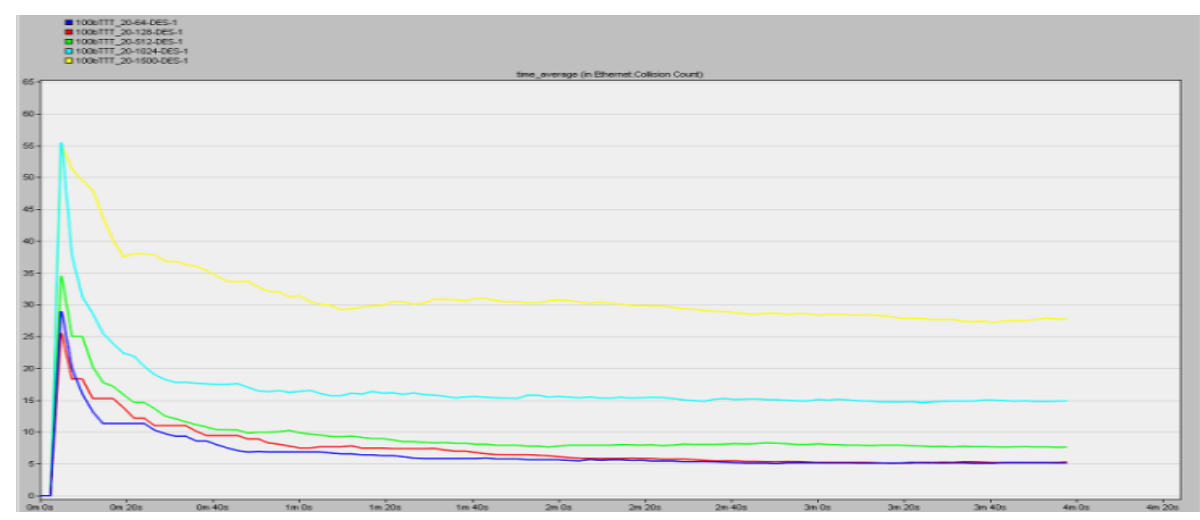

Fig. 16. Comparison between numbers of collision count at Hub2 under different frame size at 100BaseT Ethernet wiring standard

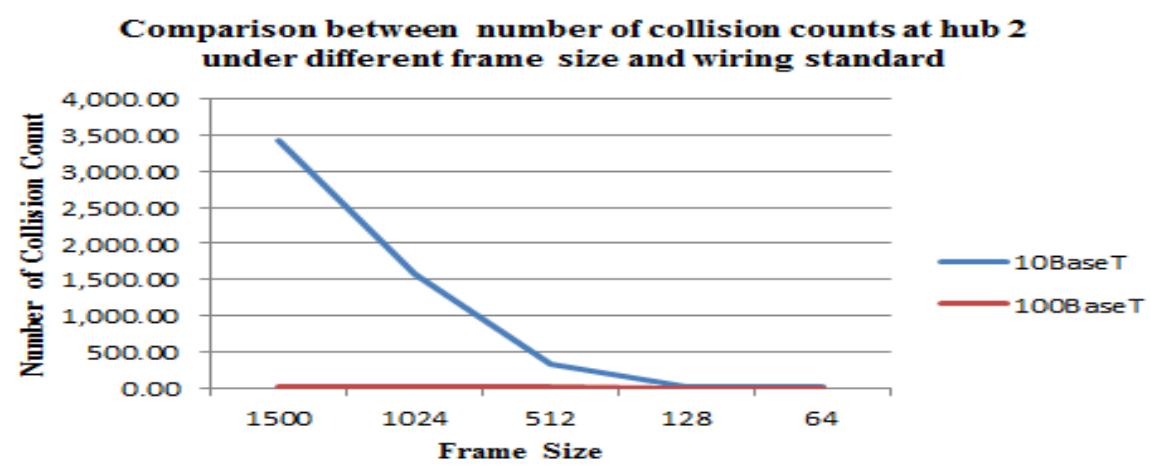

Fig. 17. Graphs for number of collision count at Hub2

Figures 15, 16 and 17 show that the number of collision count in 10BaseT is more than 100BaseT for all frame sizes.

\section{F. Utilization of Hub 2}

Table 7 Utilization of Hub2 (Avg.)

\begin{tabular}{l|l|c}
\hline \hline Time duration & \multicolumn{2}{|c}{ 4 minutes } \\
\hline \multicolumn{1}{c|}{ Devices } & \multicolumn{2}{|c}{ Hub 2 } \\
\hline \multicolumn{1}{c}{ Standards } & $\begin{array}{c}\text { 10BaseT } \\
\text { (scenario1) }\end{array}$ & $\begin{array}{c}100 \text { BaseT } \\
\text { (scenario2) }\end{array}$ \\
\hline 1500 bytes & 0.8836 & 0.0910 \\
\hline 1024 bytes & 0.6288 & 0.0626 \\
\hline 512 bytes & 0.3217 & 0.0321 \\
\hline 128 bytes & 0.092 & 0.009 \\
\hline 64 bytes & 0.053 & 0.005 \\
\hline
\end{tabular}

Table 7 shows the comparison between the collision count number at hub 2 under 10BaseT (scenario 1) and 100BaseT (scenario 2) for 1500, 1024, 512, 128 and 64 bytes of frame size. 


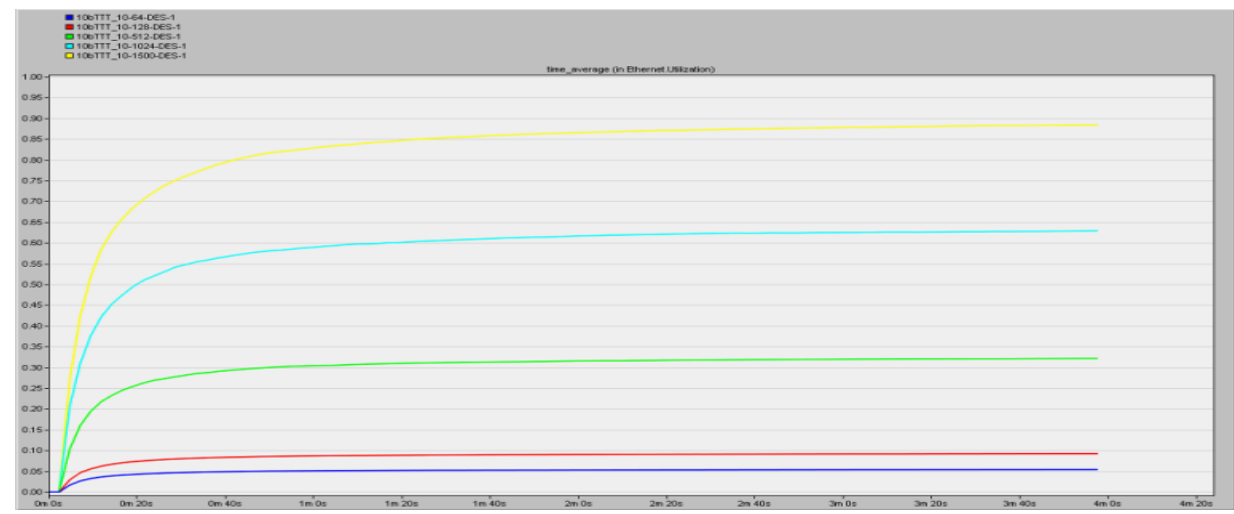

Fig. 18. Comparison between utilization at $\mathrm{Hub2}$ under different frame size at 10BaseT Ethernet wiring standard

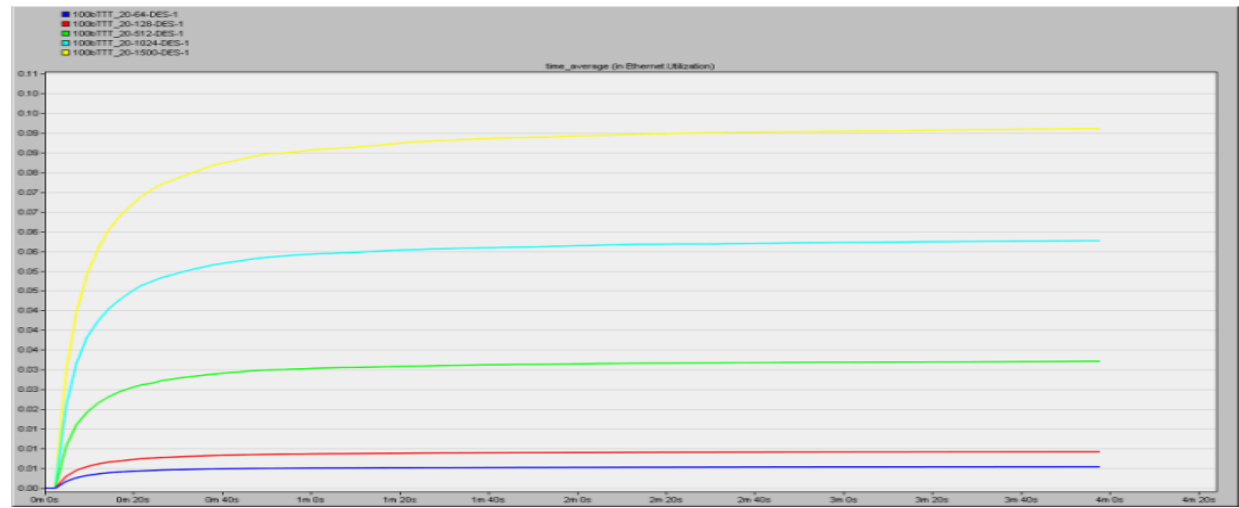

Fig. 19. Comparison between utilization at $\mathrm{Hub2}$ under different frame size at 100BaseT Ethernet wiring standard

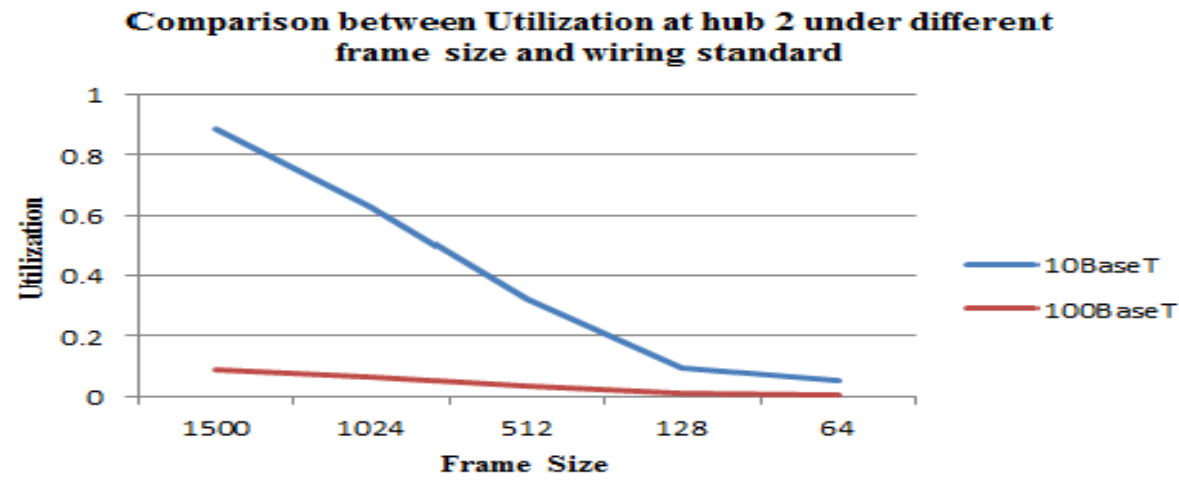

Fig. 20. Graphs for Utilization of Hub2

Figures 18, 19 and 20 illustrate that the utilization in 10BaseT is more than 100BaseT for all frame sizes. 


\section{G. Traffic forwarded (bits/sec) at Hub2}

Table 8 Traffic forwarded (bits/sec) at hub2 (Avg.)

\begin{tabular}{c|l|l}
\hline \hline Time duration & \multicolumn{2}{|c}{4 minutes } \\
\hline & \multicolumn{2}{|c}{ Traffic Forwarded (bps) } \\
\hline Devices & \multicolumn{2}{|c}{ Hub 2 } \\
\hline Standards & $\begin{array}{c}10 \text { BaseT } \\
\text { (scenario1) }\end{array}$ & \multicolumn{1}{|c}{ (scenario2) } \\
\hline 1500 bytes & $8,813,873$ & $9,105,746.133$ \\
1024 bytes & $6,276,727$ & $6,266,542.4$ \\
512 bytes & $3,214,211$ & $3,212,418.33$ \\
128 bytes & $920,963.46$ & $918,889.6$ \\
64 bytes & $538,082.4$ & $539,042.4$ \\
\hline \hline
\end{tabular}

Table 8 shows the comparison between the traffic forwarded to Hub 2 under 10BaseT (scenario1) and 100BaseT (scenario2) for 1500, 1024, 512, 128 and 64 bytes of frame size.

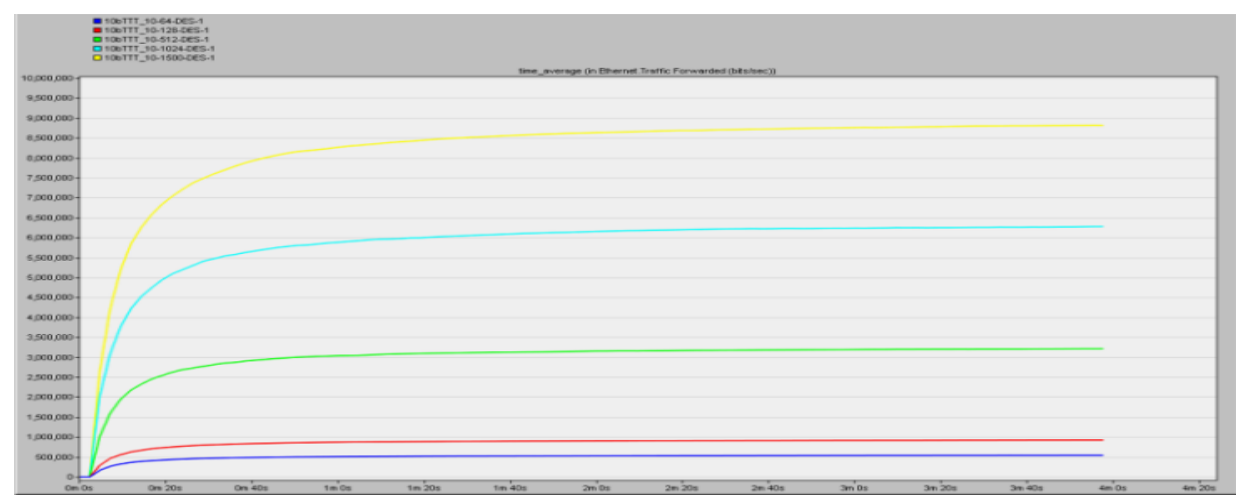

Fig. 21. Comparison between traffic forwarded (bps) at Hub2 under different frame size at 10BaseT Ethernet wiring standard.

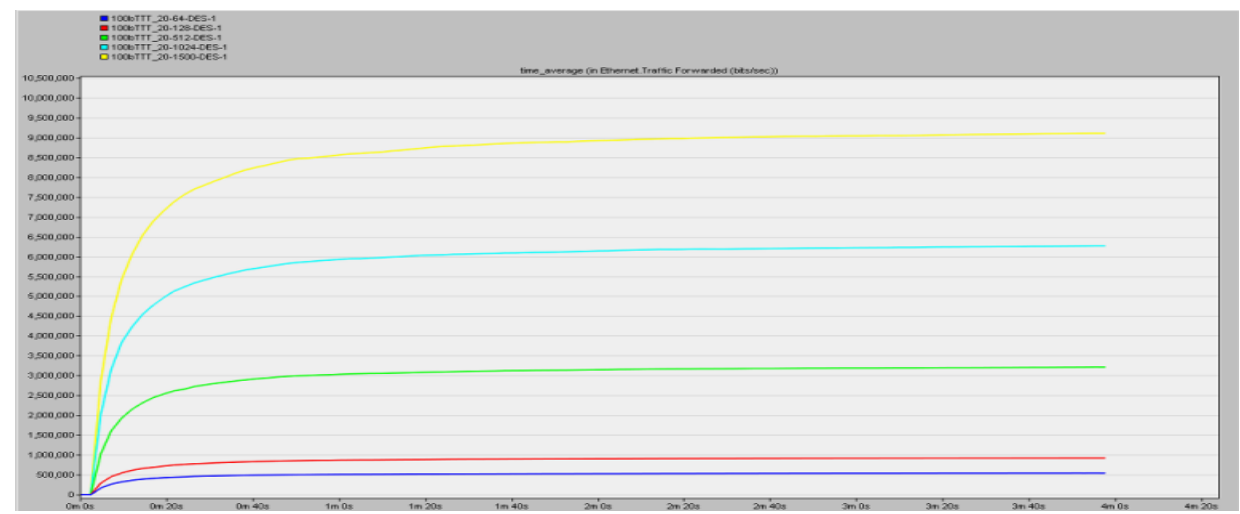

Fig. 22. Comparison between traffic forwarded (bps) at Hub2 under different frame size at 100BaseT Ethernet wiring standard 


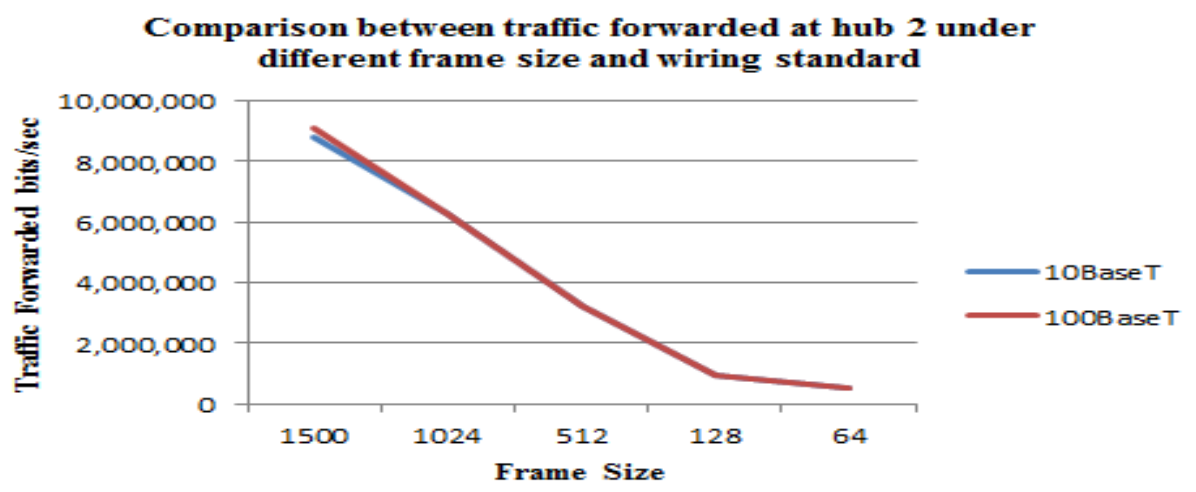

Fig. 23. Graphs of traffic forwarded to hub2 (bit/sec)

Figures 21, 22 and 23 demonstrate that at some points both curves overlap to each other, it means that traffic forwarded to hub2 is approximately same at these points.

\section{H. Traffic received (bits/sec) at hub 2}

Table 9 Traffic received (bits/sec) at hub2 (Avg.)

\begin{tabular}{c|c|c}
\hline \hline Time duration & \multicolumn{2}{|c}{ 4 minutes } \\
\hline \multicolumn{1}{c|}{ Devices } & \multicolumn{2}{|c}{ Hub 2 } \\
\hline \multicolumn{1}{c}{ Standards } & $\begin{array}{c}\text { 10BaseT } \\
\text { (scenario1) }\end{array}$ & $\begin{array}{c}\text { 100BaseT } \\
\text { (scenario2) }\end{array}$ \\
\hline 1500 bytes & $8,813,873$ & $9,105,746.133$ \\
\hline 1024 bytes & $6,276,727$ & $6,266,542.4$ \\
\hline 512 bytes & $3,214,211$ & $3,212,418.33$ \\
\hline 128 bytes & $920,963.466$ & $918,889.6$ \\
\hline 64 bytes & $538,082.4$ & $539,042.4$ \\
\hline \hline
\end{tabular}

Table 9 shows the comparison between the traffic received to Hub 2 under 10BaseT (scenario1) and 100BaseT (scenario2) for 1500, 1024, 512, 128 and 64 bytes of frame size.

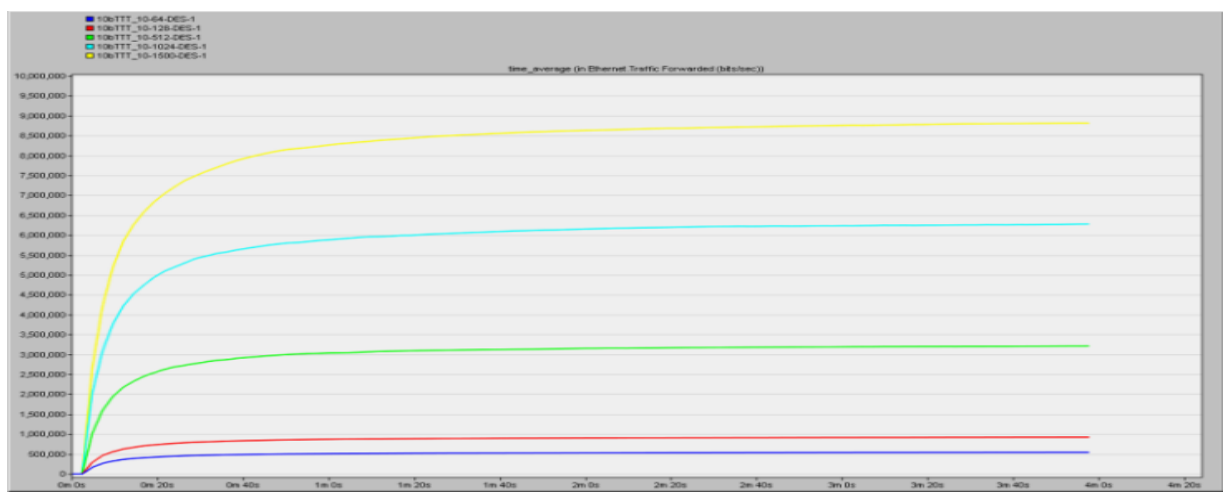

Fig. 24. Comparison between traffic received (bps) at Hub2 under different frame size at 10BaseT Ethernet wiring standard 


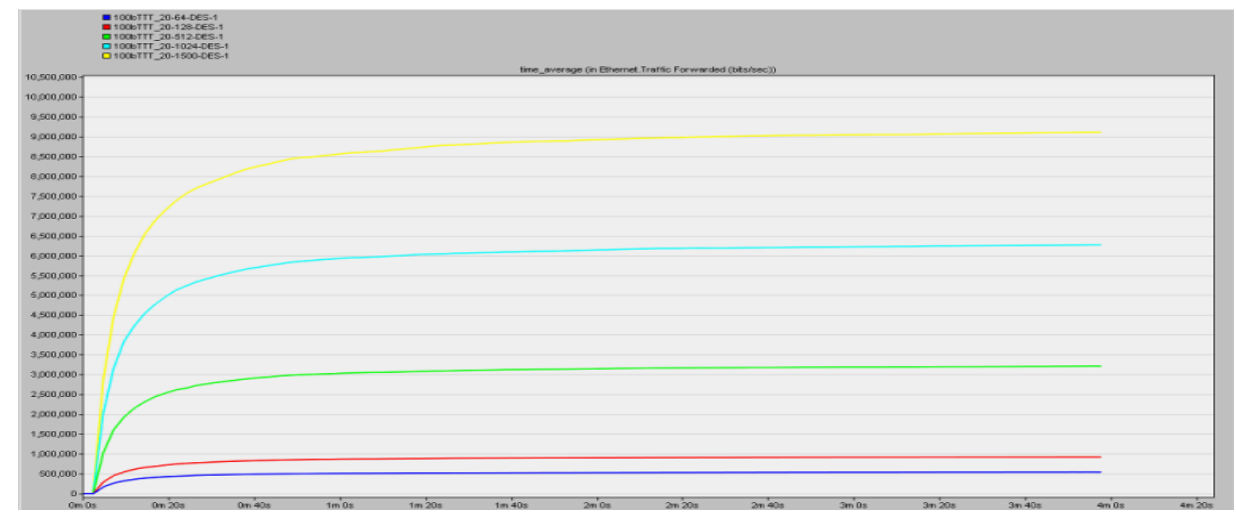

Fig. 25. Comparison between traffic received (bps) at Hub2 under different frame size at 100BaseT Ethernet wiring standard

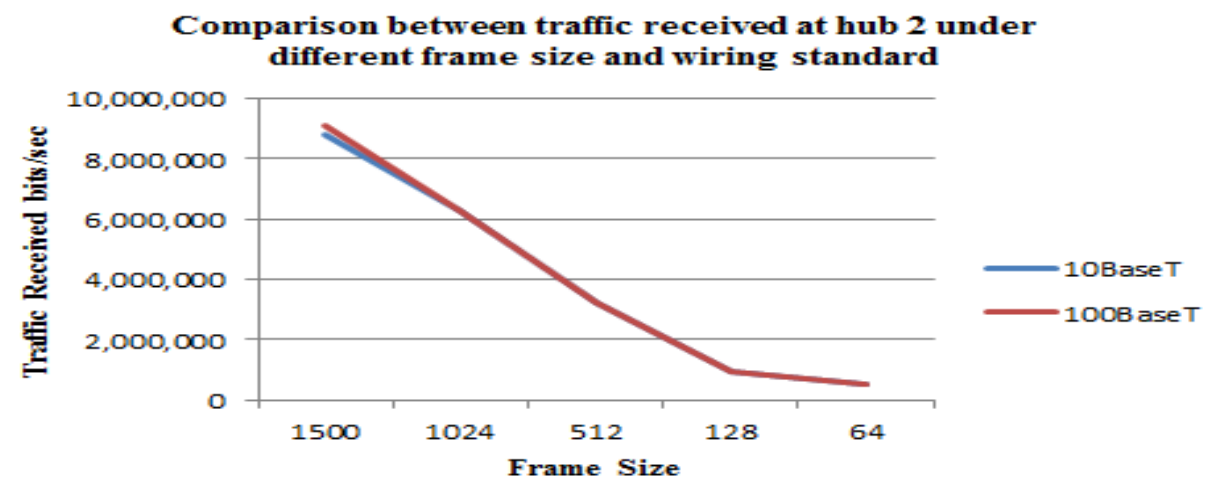

Fig. 26. Graphs of traffic received to hub2 (bit/sec)

Figures 24, 25 and 26 show that at some points both curves overlap to each other; it means that traffic received at hub2 is approximately same at these points.

\section{Traffic forwarded (bits/sec) at switch}

Table 10 Traffic forwarded (bits/sec) to switch (Avg.)

\begin{tabular}{c|l|l}
\hline \hline Time duration & \multicolumn{2}{|c}{ 4 minutes } \\
\hline Devices & \multicolumn{2}{|c}{ Traffic Forwarded (bps) } \\
\hline Standards & $\begin{array}{c}\text { 10BaseT } \\
\text { (scenario1) }\end{array}$ & $\begin{array}{c}\text { 100BaseT } \\
\text { (scenario2) }\end{array}$ \\
\hline 1500 bytes & $6,091,588.533$ & $6,281,372.066$ \\
1024 bytes & $4,312,105$ & $4,327,995$ \\
512 bytes & $2,212,381.533$ & $2,220,971.6$ \\
128 bytes & 634,480 & $633,561.133$ \\
64 bytes & 370,812 & 372,297 \\
\hline \hline
\end{tabular}

Table 10 shows the comparison between the traffic forwarded to switch under 10BaseT (scenario1) and 100BaseT (scenario2) for 1500, 1024, 512, 128 and 64 bytes of frame size. 


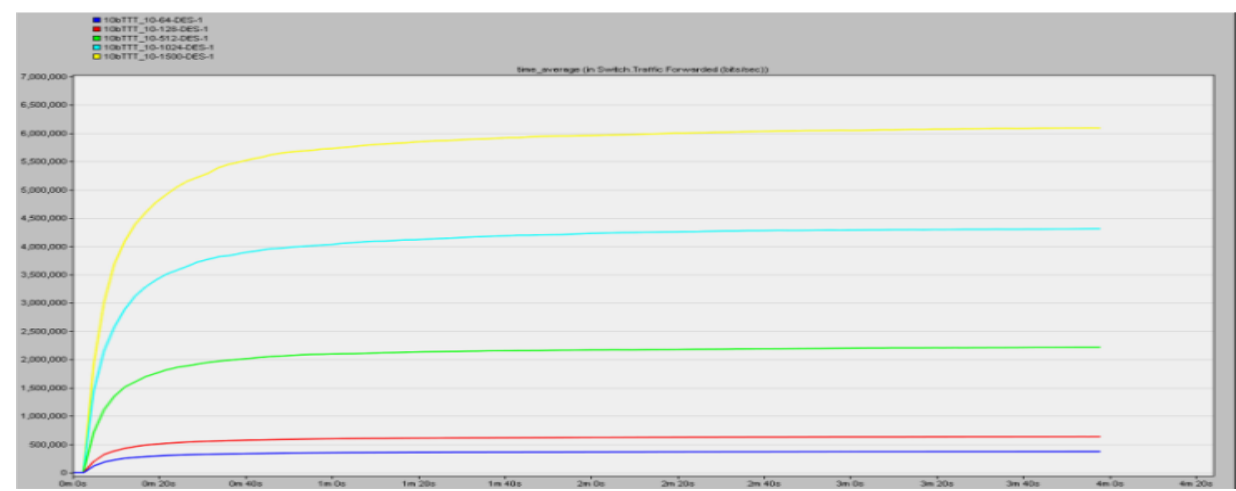

Fig. 27. Comparison between traffic forwarded (bps) at Switch under different frame size at 10BaseT Ethernet wiring standard

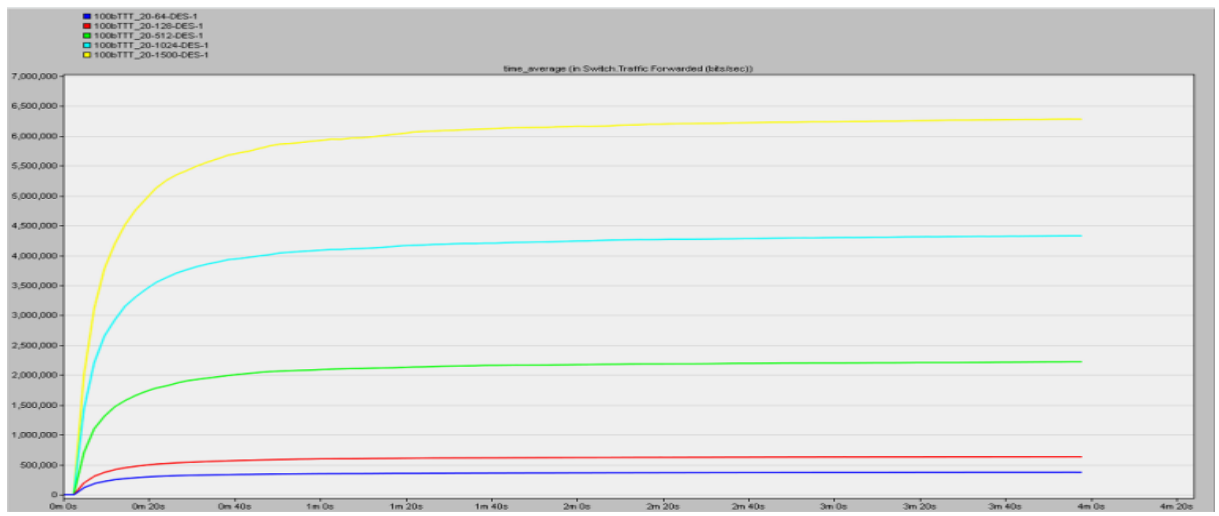

Fig. 28. Comparison between traffic forwarded (bps) at Switch under different frame size at 100BaseT Ethernet wiring standard

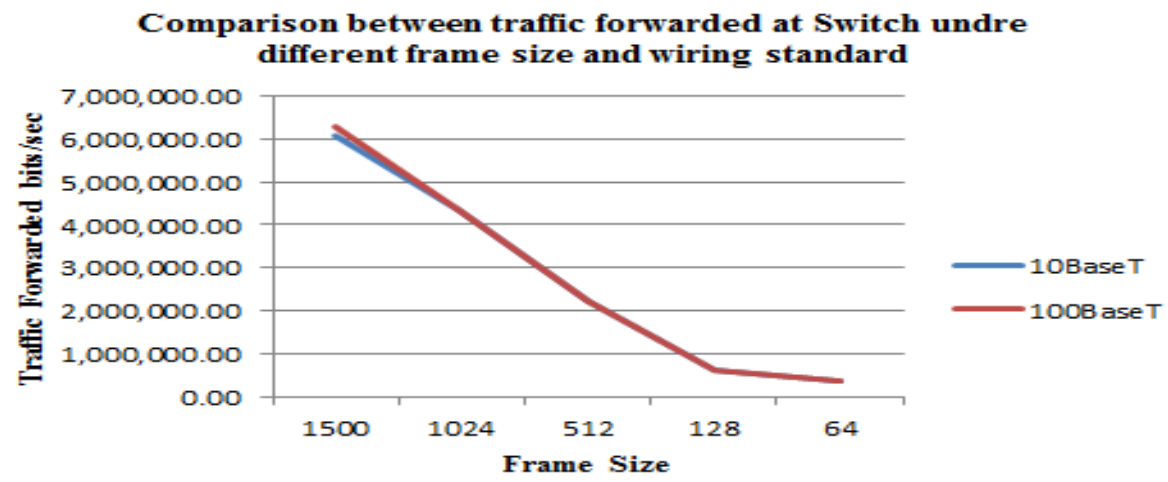

Fig. 29. Graphs of traffic forwarded to switch (bit/sec)

J. Traffic received (bits/sec) at switch 
Table 11 Traffic Received (bits/sec) to switch (Avg.)

\begin{tabular}{c|l|l}
\hline \hline Time duration & \multicolumn{2}{|c}{ 4 minutes } \\
\hline & \multicolumn{2}{|c}{ Traffic Received (bps) } \\
\hline Devices & \multicolumn{2}{|c}{ Switch } \\
\hline Standards & $\begin{array}{c}\text { 10BaseT } \\
\text { (scenario1) }\end{array}$ & \multicolumn{1}{|c}{$\begin{array}{c}\text { 100BaseT } \\
\text { (scenario2) }\end{array}$} \\
\hline 1500 bytes & $11,559,450$ & $11,924,876.13$ \\
1024 bytes & $8,223,810$ & $8,201,585$ \\
512 bytes & $4,211,356$ & $4,205,994.33$ \\
128 bytes & $1,206,466.8$ & $1,203,874.466$ \\
64 bytes & 705,867 & 705,708 \\
\hline \hline
\end{tabular}

Table 11 shows the comparison between the traffic received by switch under 10BaseT (scenario1) and 100BaseT (scenario2) for 1500, 1024, 512, 128 and 64 bytes of frame size.

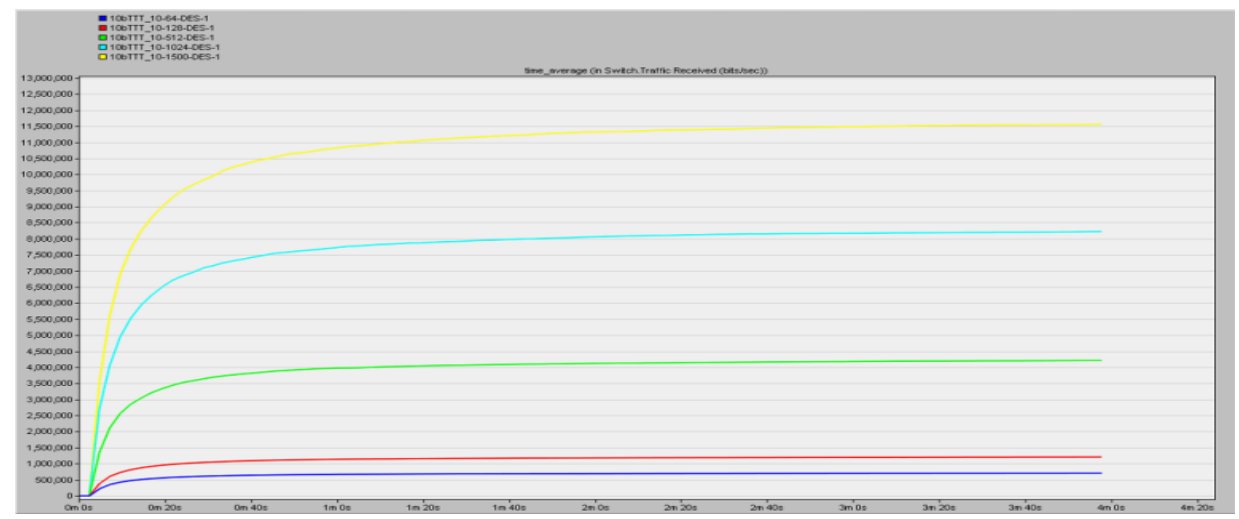

Fig. 30. Comparison between traffic received (bps) at switch under different frame size at 10BaseT Ethernet wiring standard

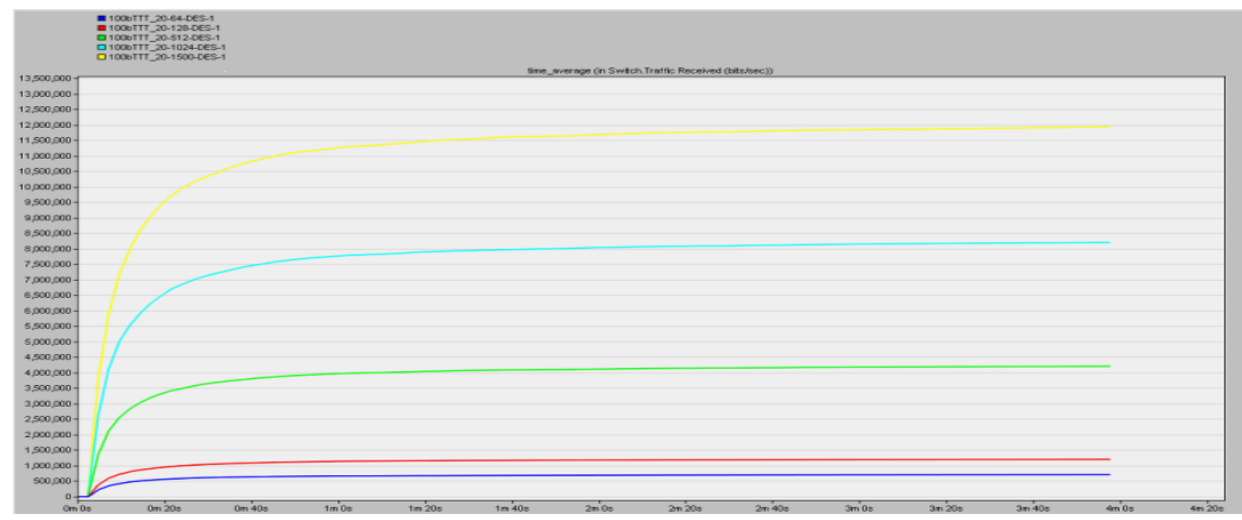

Fig. 31. Comparison between traffic received (bps) at switch under different frame size at 100BaseT Ethernet wiring standard 


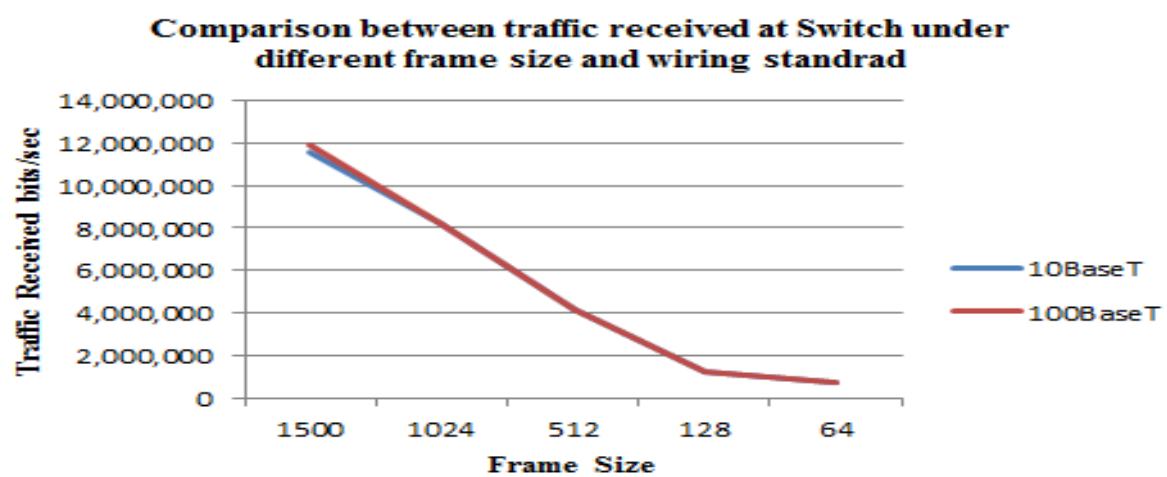

Fig. 32. Graphs of traffic received at switch (bit/sec)

\section{K. Traffic filtered (bits/sec) by switch}

Table 12 Traffic filtered (bits/sec) to switch (Avg.)

\begin{tabular}{l|l|l}
\hline \hline Time duration & \multicolumn{2}{|l}{4 minutes } \\
\hline & \multicolumn{2}{|l}{ Traffic Filtered (bps) } \\
\hline Devices & Switch & \\
\hline Standards & $\begin{array}{l}\text { 10BaseT } \\
\text { (scenario1) }\end{array}$ & $\begin{array}{l}\text { 100BaseT } \\
\text { (scenario2) }\end{array}$ \\
\hline 1500 bytes & $5,467,861.67$ & $5,643,504.04$ \\
1024 bytes & $3,911,705$ & $3,873,590$ \\
512 bytes & $1,998,974.8$ & $1,985,022.7$ \\
128 bytes & $571,986.8$ & $570,313.33$ \\
64 bytes & 335,055 & 333,411 \\
\hline
\end{tabular}

Table 12 shows the comparison between the traffic received by switch under 10BaseT (scenario1) and 100BaseT (scenario2) for 1500, 1024, 512, 128 and 64 bytes of frame size.

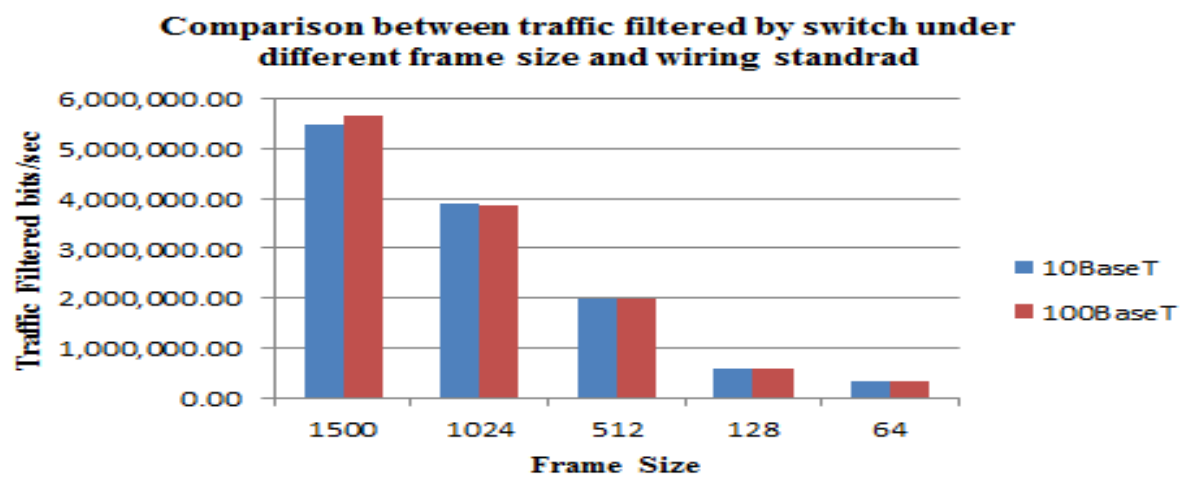

Fig. 33. Comparison between the traffic filtered by switch under different frame size and wiring standard

Figure 33 shows that the initially filtered traffic is better in case of 100BaseT for 1500 bytes frame size than 10BaseT. For 1024, 512, 128 and 64 bytes frame size the switch under 10BaseT filtered more traffic than 100BaseT, it means that the performance of 10BaseT Ethernet wiring standard is become better at the case of low traffic data 


\section{Conclusions}

The performance of LANs under different Ethernet wiring standard having different frame size are compared and the observations from our simulations outcomes are:

a) The number of collision counts in 10BaseT is always more than 100BaseT for all the frame sizes because of the nature of 10BaseT [5], [9] and [10]

b) Hubs are more utilized in case of 10BaseT because of the large value of collision count so the more retransmission attempts will be required.

c) The performance of a switch is better for 100BaseT wiring standard at the case of 1500 bytes frame size than that the case of 10BaseT because it filters more traffic. When the frame size is 1024 bytes, filtered traffic will be approximately the same for both 10BaseT and 100BaseT. When frame size is further reduced to 512, 128 and 64 the results show that the performance of 10BaseT becomes better than 100BaseT because the switch filtered more traffic than 100BaseT.

d) If we have LAN in which high traffic is not required and the frame size will be fixed to 512,128 or 64 bytes, 10BaseT will give us good result and performs better as compared to 100BaseT Ethernet wiring standard.

e) At the case of small frame size we will not able to transfer more traffic per seconds (traffic receiving and forwarding is less) so we cannot use them in heavy traffic (refer tables 10 and 11).

\section{References}

[1] Mohammad Wazid Roshan Singh Sachan, R.H. Goudar "Analysis of a LAN under Different Ethernet Wiring Standards with Variation in Time and Components", UACEE International Journal of Advances in Computer Networks and its Security - Volume 2: Issue 3 [ISSN 2250 - 3757]

[2] Mohammad Wazid, Roshan Singh Sachan, R.H. Goudar "Performance Evaluation of a LAN under Different Ethernet Wiring Standards with Different Frame Size", International Journal of Computer Applications (0975 - 8887) Volume 43- No.13, April 2012.

[3] A. Forouzan, "Data Communication \& Networking" 4th Edition 2006: Tata McGraw Hill.

[4] William Stallings, "Data and Computer Communications" 8th Edition 2006: Pearson Education.

[5] Charles Spurgeon and Chuck Toporek, "Ethernet: The Definitive Guide", 1st Edition 2000: O’Reilly \& Associates.

[6] Todd Lammle, "Cisco Certified Network Associate Study Guide" 2nd Edition 2000: SYBEX, Inc., Alameda, CA.

[7] Daniel Minoli, Peter Johnson and Emma Minoli, "Ethernet-based Metro Area Networks", 1st Edition 2002: McGraw-Hill.

[8] Xinjie Chang, "Network simulations with OPNET", IEEE Conference Publications of Simulation Conference Proceedings, 1999 winter.

[9] S. Kabir, S. Khatun, M. K. Abdullah, M.A. Mahdi and S. B. A. Anas, "Throughput Analysis of an Enhanced CSMA/CD Based Single Channel Fast Ethernet Optical 
LAN", International Conference on Advance Communication Technology (ICACT) 2005.

[1] R. M. Daud, H. M. Elsayed and H. H. Amer, "Performance of Fast and Gigabit Ethernet in Networked Control System", 46th IEEE International Midwest Symposium on Circuit and System 2003. 